\title{
$\mathrm{MIMO}$ 검출기에 적용 가능한 저 복잡도 복합 $\mathrm{QR}$ 분해 구조
}

\section{A Low-complexity Mixed QR Decomposition Architecture for MIMO Detector}

\author{
신 동 엽*, 김 철 우*, 박 종 선 ${ }^{* \star}$ \\ Dongyeob Shin*, Chulwoo Kim*, Jongsun Park ${ }^{* \star}$
}

\begin{abstract}
This paper presents a low complexity QR decomposition (QRD) architecture for MIMO detector. In the proposed approach, various CORDIC-based QRD algorithms are efficiently combined together to reduce the computational complexity of the QRD hardware. Based on the computational complexity analysis on various QRD algorithms, a low complexity approach is selected at each stage of QRD process. The proposed QRD architecture can be applied to any arbitrary dimension of channel matrix, and the complexity reduction grows with the increasing matrix dimension. Our QR decomposition hardware was implemented using Samsung $0.13 \mu$ $\mathrm{m}$ technology. The numerical results show that the proposed architecture achieves $47 \%$ increase in the QAR (QRD Rate/Gate count) with $28.1 \%$ power savings over the conventional Householder CORDIC-based architecture for the $4 \times 4$ matrix decomposition.

$$
\text { 요 약 }
$$

본 논문에서는 $\mathrm{MIMO}$ 검출기를 위한 저 복잡도 $\mathrm{QR}$ 분해 구조를 제시한다. 제안된 접근 방식에서는, $\mathrm{QRD}$ 하드 웨어의 연산 복잡도를 감소시키기 위해 다양한 코딕 기반 $\mathrm{QRD}$ 알고리즘들이 효율적으로 조합된다. 다양한 $\mathrm{QRD}$ 알고리즘들에 대한 연산 복잡도 분석에 기초하여, $\mathrm{QRD}$ 과정의 매 단계마다 저 복잡도 접근 방식이 선택된다. 제 안된 $\mathrm{QRD}$ 구조는 어떤 임의의 차원을 갖는 채널 매트릭스에도 적용 될 수 있고, 매트릭스 차원의 증가에 따라 연산 복잡도 감소도 늘어난다. 제안하는 $\mathrm{QR}$ 분해 하드웨어는 삼성 $0.13 \mu \mathrm{m}$ 공정을 사용하여 구현되었다. 실험결과, $4 \times 4$ 행렬의 $\mathrm{QR}$ 분해에 대한 제안 구조는 기존의 Householder 코딕 기반의 구조에 비해 $47 \%$ 의 $\mathrm{QAR}(\mathrm{QRD}$ Rate/Gate count) 향상과 $28 \%$ 의 전력을 절감을 이뤄낼 수 있었다.
\end{abstract}

Key words : QR Decomposition, MIMO, Givens Rotation, Householder, Multidimensional CORDIC

\section{I. 서론}

Multiple-input and multiple-output (MIMO) 시스

* Dept. of Electrical Engineering, Korea University

$\star$ Corresponding author

jongsun@korea.ac.kr, 02-3290-3669

※ Acknowledgment

This work is supported by National Research Foundation (NRF) Grant funded by the Korea Government (MEST) (No. 2012-0020128).

Manuscript received Mar. 14, 2014; revised Mar. 24, 2014 ; accepted Mar 24. 2014
템은 높은 주파수 효율과 링크 안정성 때문에 IEEE $802.11 \mathrm{ac}$ (WLAN)과 같은 표준이나 long term evolution (LTE) 등의 어플리케이션에서 널리 채택되 고 있다[1]. 복잡한 $\mathrm{MIMO}$ 알고리즘에 기반한 연산이 모바일 기기에서 수행되기 위해서, $\mathrm{MIMO}$ 수신기의 저전력·고속 VLSI 구현이 중요한 관심사가 되어 왔 다. $\mathrm{MIMO}$ 수신기의 핵심 연산 중 하나는 $\mathrm{MIMO}$ detector에서 필요한 상삼각 $\mathrm{R}$ 행렬의 생성을 위한 삼각화 연산이라고 할 수 있다 [2-3]. QR 분해는 높 은 안정성을 갖는 특성과 least square solution을 찾 는 능력으로 인해 역행렬 도출이나 행렬의 삼각화를 수행하는데 널리 사용되어 왔다 [4]. Adavanced 4G 
무선 통신 표준들은 높은 처리량과 많은 안테나 배치 를 갖는 $\mathrm{MIMO}$ 시스템들을 필요로 하는데, 이를 위 해서는 복잡한 $\mathrm{QR}$ 분해가 매우 자주 수행되어야 한 다. $\mathrm{QRD}$ 하드웨어에 대한 대부분의 이전 연구들은 이런 MIMO 시스템의 증가한 data rate를 만족시키기 위해 하드웨어 속도를 향상시키는 것에 집중해왔고, 그 결과 큰 하드웨어 면적과 전력 소모가 필요해졌 다. 하지만, 높은 속도를 유지하면서 저 복잡도와 저 전력을 소모하는 $\mathrm{QRD}$ 하드웨어의 구현 또한 휴대용 통신 기기의 배터리 수명 증가를 위해서는 반드시 고 려해야 할 사항이다.

Coordinate rotation digital computer (CORDIC) 은 덧셈기와 쉬프터만으로 구현이 가능하기 때문에 $\mathrm{QRD}$ 하드웨어에 흔하게 사용되어 왔다 [5]. 코딕 기반의 $\mathrm{QRD}$ 하드웨어 구현에는 기븐스 회전(GR) 알고리즘 이 가장 널리 사용된다. 하지만, 기븐스 회전은 일반 적으로 순차적인 zero insertion 동작으로 인해 높은 대기시간을 갖는데 [6], 이는 향상된 무선 통신 표준 의 요구사항을 충족시키는데 장애물이 된다. 이 문제 를 극복하기 위하여, zero insertion의 병렬 처리 정도 를 증가 시킨 고속 기븐스 회전 기반 $\mathrm{QRD}$ 구조가 제 안되었으나 [7-8], 기븐스 회전 알고리즘을 이용한 병 렬 zero insertion은 큰 연산 복잡도를 유발한다[8]. 고속 $\mathrm{QRD}$ 하드웨어의 다른 해결 방법으로 하우스홀 더 $(\mathrm{HH})$ 코딕 알고리즘과[9-10] 다차원 $(\mathrm{MD})$ 코딕 알 고리즘[11] 또한 제안되었다. 고속 하우스홀더 혹은 다차원 기반 $\mathrm{QRD}$ 는 고속 기븐스 회전 기반의 $\mathrm{QRD}$ 와 비교하여 상대적으로 작은 복잡도를 가지지만[8], 그럼에도 불구하고 고속 $\mathrm{QRD}$ 의 하드웨어 오버헤드는 여전히 매우 크다.

본 논문에서는, 여러 코딕 기반 $\mathrm{QR}$ 분해 알고리즘 들을 고속-저복잡도 관점에서 조합하여 각각의 열에 대한 zero insertion 동작들을 동시에 수행하는 저 복 잡도 $\mathrm{QRD}$ 구조를 제안한다.

\section{II. 기존 코딕 기반 $\mathrm{QR}$ 분해 알고리즘}

$\mathrm{N}_{\mathrm{t}}$ 개의 전송 안테나와 $\mathrm{N}_{\mathrm{r}}$ 개의 수신 안테나를 포함하 는 공간 다중 방식 MIMO 시스템을 고려하면, 이산 시간의 수신 신호, $\mathrm{y}$ 는 아래와 같이 표현할 수 있다.

$$
y=H x+n
$$

수식 (1)에서 $\mathrm{y}$ 는 $\left(\mathrm{N}_{\mathrm{r}} \times 1\right)$ 사이즈의 수신 신호 벡 터, $\mathrm{x}$ 는 $\left(\mathrm{N}_{\mathrm{t}} \times 1\right)$ 사이즈의 전송 신호 벡터이고, $\mathrm{H}$ 는 $\left(\mathrm{N}_{\mathrm{r}} \times \mathrm{N}_{\mathrm{t}}\right)$ 사이즈의 복소 채널 행렬, $\mathrm{n}$ 은 $\left(\mathrm{N}_{\mathrm{r}} \times 1\right)$ 사
이즈의 AWGN 벡터이다. $\mathrm{QR}$ 분해는 복소 채널 행렬 $\mathrm{H}$ 를 직교 행렬 $\mathrm{Q}$ 와 상삼각행렬 $\mathrm{R}$ 의 곱으로 분해하 는 과정이며, 이는 다음과 같이 표기할 수 있다.

$$
H=Q R
$$

$\mathrm{H}$ 의 왼쪽에 역행렬 $\mathrm{Q}^{-1}$ 을 곱함으로써, 상삼각행렬 $\mathrm{R}$ 은 (3)과 같이 표현할 수 있다. $\Theta_{\mathrm{i}}$ 가 해당하는 복소 성분을 나타낼 때 $2 \times 2$ 복소행렬이 (4)와 같다고 한 다면, $\mathrm{Q}^{-1}$ 은 3 개 행렬들의 곱으로 나타내어질 수 있 다. 코딕 기반의 $\mathrm{QRD}$ 과정에서는 각각의 행렬 $\left(\mathrm{Q}_{\mathrm{RT} .1}{ }^{-1}, \mathrm{Q}_{\mathrm{ZI.1}}{ }^{-1}, \mathrm{QRT}_{2}{ }^{-1}\right)$ 의 곱셈을 반복적으로 수행한 다.

$$
\begin{aligned}
& \mathbf{R}=\mathbf{Q}^{-1} \mathbf{H}=\left(\mathbf{Q}_{R T .2}^{-1} \cdot \mathbf{Q}_{Z I .1}^{-1} \cdot \mathbf{Q}_{R T .1}^{-1}\right) \mathbf{H} \\
& \mathbf{H}=\left[\begin{array}{ll}
\mathbf{h}_{11} & \mathbf{h}_{12} \\
\mathbf{h}_{21} & \mathbf{h}_{22}
\end{array}\right]=\left[\begin{array}{ll}
\left|h_{11}\right| e^{j \theta_{11}} & \left|h_{12}\right| e^{j \theta_{12}} \\
\left|h_{21}\right| e^{j \theta_{21}} & \left|h_{22}\right| e^{j \theta_{22}}
\end{array}\right]
\end{aligned}
$$

$\mathrm{Q}_{\mathrm{RT} .1}{ }^{-1}, \mathrm{Q}_{\mathrm{RT} .2}{ }^{-1}$ 은 (5)에서 표현된 것과 같이 다른 알 고리즘들에 대해서도 같은 방법으로 생성되지만, 행 렬 $\mathrm{QZZI}^{-1}$ 은 (6)과 (7)에서 나타나듯이 기븐스 회전과 하우스홀더 알고리즘에서 각각 다르게 생성된다. 여 기서 $\mathrm{n}$ 은 코딕 반복(iteration)의 인덱스를 뜻한다.

$$
\mathbf{Q}_{R T .1}^{-1}=\left[\begin{array}{cc}
e^{-j \theta_{11}} & 0 \\
0 & e^{-j \theta_{21}}
\end{array}\right], \quad \mathbf{Q}_{R T .2}^{-1}=\left[\begin{array}{cc}
1 & 0 \\
0 & e^{-j \theta_{22}^{*}}
\end{array}\right]
$$

$$
\mathbf{Q}_{Z I .1(G R)}^{-1}=\prod \frac{1}{\sqrt{1+2^{-2 n}}}\left[\begin{array}{cc}
1 & 2^{-n} \operatorname{sign}\left(h_{21}^{*} n\right) \\
-2^{-n} \operatorname{sign}\left(h_{21}^{*}\right) & 1
\end{array}\right]
$$

$$
\mathbf{Q}_{Z Z 1(H H)}^{-1}=\prod \frac{1}{1+2^{-2 n}}\left[\begin{array}{cc}
1-2^{-2 n} & 2^{-(n-1)} \cdot \operatorname{sign}^{n} \\
-2^{-(n-1)} \cdot \operatorname{sign}^{n} & 1-2^{-2 n}
\end{array}\right]
$$

여기서 $\Theta_{\mathrm{i}}{ }^{*}$ 는 $\mathrm{QZZI}^{-1} \cdot \mathrm{QRT}_{\mathrm{RT} .}^{-1} \cdot \mathrm{H}$ 의 상응하는 복소 성분을 나타내고, $\mathrm{h}_{\mathrm{i}}^{* \mathrm{n}}$ 은 $\mathrm{n}$ 번째 코딕 반복에서 $\mathrm{QZZI}^{-1}$ 곱셈의 $\mathrm{i}$ 위치의 복소 성분을 나타내며, $\operatorname{sign}^{\mathrm{n}}$ 은 $\mathrm{n}$ 번 째 코딕 반복의 $\mathrm{h}^{*}{ }_{11}{ }^{\mathrm{n}} \cdot \mathrm{h}^{*}{ }^{*}{ }^{\mathrm{n}}$ 의 부호를 나타내고, $\theta_{1}$ 은 $\tan \left(\left|\mathrm{h}_{21}\right| /\left|\mathrm{h}_{11}\right|\right)$ 와 같다. 일반적으로 코딕 기반의 $\mathrm{QRD}$ 알고리즘들은 하드웨어 구현에서 각각 나름의 이점을 갖고 있다. $2 \times 2$ 채널 매트릭스의 $\mathrm{QRD}$ 과정에서, 
하우스홀더 알고리즘이 $\mathrm{Q}_{\text {ZI.1 }}^{-1}$ 의 대각 성분에 추가적 인 뺄셈 연산을 갖고 있으므로 연산 복잡도를 감소시 키기 위해서는 기븐스 회전 알고리즘이 하우스홀더 알고리즘 보다 적절한 선택이다. 반대로 하우스홀더 알고리즘은 $\mathrm{k}>2$ 인 $\mathrm{k} \times \mathrm{k}$ 행렬에서 처리량 관점으로 볼 때 기븐스 회전과 비교하여 장점이 있다[11].

\section{III. 제안하는 복합 $\mathrm{QR}$ 분해 방법}

큰 공간 차원을 갖는 $\mathrm{MIMO}$ 시스템을 위한 저 복 잡도 고속 $\mathrm{QRD}$ 하드웨어 설계를 위하여, 본 논문에 서는 다양한 코딕 기반의 $\mathrm{QRD}$ 알고리즘들을 최적으 로 조합하는 $\mathrm{QRD}$ 방식을 제안한다. 제안하는 방법 을 위한 사례 연구를 위해 $4 \times 4$ 복소 행렬을 $\mathrm{H}$ 라고 가정하자. 일반적인 코딕 기반 $\mathrm{QRD}$ 과정은 (8)부터 (11) 처럼 표현할 수 있다. (8)부터 (10)에는 첫 번째, 두 번째 그리고 세 번째 열의 zero insertion 과정이 나타나 있다. 여기서 행렬 $\mathrm{Q}_{\mathrm{RT} .1}{ }^{-1}, \mathrm{QRT}_{\mathrm{R} .2}{ }^{-1}, \mathrm{Q}_{\mathrm{RT} .3}{ }^{-1}$ 는 $\mathrm{QRD}$ 알고리즘에 관계없이 유일하게 생성된다. 반면 에, 행렬 $\mathrm{QZII}^{-1}, \mathrm{QZI}^{-1}{ }^{-1}, \mathrm{Q}_{\text {ZI.3 }}{ }^{-1}$ 는 먼저 $\mathrm{QRD}$ 알고리즘 이 각 열에 대해 정해지면, 정해진 $\mathrm{QRD}$ 알고리즘에 따라 다른 방식으로 생성된다.

$$
\begin{aligned}
& \mathbf{H}_{1}=\left(\mathbf{Q}_{Z Z .1}^{-1} \cdot \mathbf{Q}_{R T .1}^{-1}\right) \mathbf{H}=\left[\begin{array}{cccc}
\left|h_{11}^{1}\right| & h_{12}^{1} & h_{13}^{1} & h_{14}^{1} \\
0 & h_{22}^{1} & h_{23}^{1} & h_{24}^{1} \\
0 & h_{32}^{1} & h_{33}^{1} & h_{34}^{1} \\
0 & h_{42}^{1} & h_{43}^{1} & h_{44}^{1}
\end{array}\right] \\
& \mathbf{H}_{2}=\left(\mathbf{Q}_{Z Z .2}^{-1} \cdot \mathbf{Q}_{R T .2}^{-1}\right) \mathbf{H}_{1}=\left[\begin{array}{cccc}
h_{11}^{1} \mid & h_{12}^{1} & h_{13}^{1} & h_{14}^{1} \\
0 & \left|h_{22}^{2}\right| & h_{23}^{2} & h_{24}^{2} \\
0 & 0 & h_{33}^{2} & h_{34}^{2} \\
0 & 0 & h_{43}^{2} & h_{44}^{2}
\end{array}\right]
\end{aligned}
$$$$
\mathbf{H}_{3}=\left(\mathbf{Q}_{\mathrm{Z} J 3}^{-1} \cdot \mathbf{Q}_{R T .3}^{-1}\right) \mathbf{H}_{2}=\left[\begin{array}{cccc}
\left|h_{11}^{1}\right| & h_{12}^{1} & h_{13}^{1} & h_{14}^{1} \\
0 & \left|h_{22}^{2}\right| & h_{23}^{2} & h_{24}^{2} \\
0 & 0 & h_{33}^{3} \mid & h_{34}^{3} \\
0 & 0 & 0 & h_{44}^{3}
\end{array}\right]
$$

$$
\mathbf{R}=\left(\mathbf{Q}_{R T .4}^{-1}\right) \mathbf{H}_{3}=\left[\begin{array}{cccc}
\left|h_{11}^{1}\right| & h_{12}^{1} & h_{13}^{1} & h_{14}^{1} \\
0 & \left|h_{22}^{2}\right| & h_{23}^{2} & h_{24}^{2} \\
0 & 0 & \left|h_{33}^{3}\right| & h_{34}^{3} \\
0 & 0 & 0 & \left|h_{44}^{4}\right|
\end{array}\right]
$$

예를 들어, 각각 하우스홀더와 다차원 알고리즘으로 생성된 코딕 반복의 $\mathrm{i}$ 번째 행렬 $\mathrm{QZII}^{-1}$ 이 (12)와 (13) 에 나타나 있다. 이와 유사하게, 행렬 $\mathrm{QZII}^{-1}$ 와 $\mathrm{QZZ}^{-1}$ 도 $\mathrm{QRD}$ 알고리즘이 기븐스 회전, 하우스홀더, 다차원 중 어느 것인지에 따라 다른 방식으로 정해진다 [8-10].

기존 $\mathrm{QRD}$ 방식의 가장 큰 문제점은 $\mathrm{QRD}$ 알고리 즘이 정해짐에 따라 행렬 $\mathrm{QZII}^{-1}, \mathrm{Q}_{\mathrm{ZI} .2^{-1}}, \mathrm{Q}_{\mathrm{ZI} .3^{-1}}$ 이 정해 진 알고리즘에 의해 아무 고려 없이 결정된다는 것이 다. 본 논문에서는, 이런 문제점을 해결하기 위해 다 양한 코딕 기반 $\mathrm{QRD}$ 알고리즘들에 의해 생성되는 행 렬 $\mathrm{QZII}^{-1}, \mathrm{QZII}^{-1}, \mathrm{QZI}^{-1}{ }^{-1}$ 의 연산 과정을 연산 복잡도와 대기시간 측면에서 분석하였다. $\mathrm{N}$ 이 코딕 반복의 횟 수를 나타내고, $\mathrm{M}=\mathrm{N}(\mathrm{N}+1)$ 일 때, 3 가지 다른 연

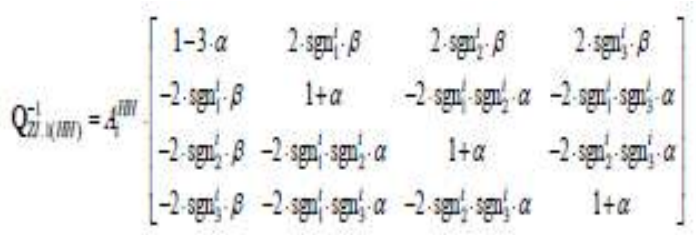

$\mathrm{Q}_{z \perp(M \omega)}^{-1}=A_{1}^{M D}\left[\begin{array}{cccc}1 & -\operatorname{sgn}_{1}^{\prime} \cdot \beta & -\operatorname{sgn}_{2}^{\prime} \cdot \beta & -\operatorname{sgn}_{3}^{\prime} \cdot \beta \\ \operatorname{sgn}_{1}^{\prime} \cdot \beta & 1 & \operatorname{sgn}_{3}^{\prime} \cdot \beta & -\operatorname{sgn}_{2}^{t} \cdot \beta \\ \operatorname{sgn}_{2}^{\prime} \cdot \beta & -\operatorname{sgn}_{3}^{\prime} \cdot \beta & 1 & \operatorname{sgn}_{1}^{\prime} \cdot \beta \\ \operatorname{sgn}_{3}^{\prime} \cdot \beta & \operatorname{sgn}_{2}^{\prime} \cdot \beta & -\operatorname{sgn}_{1}^{\prime} \cdot \beta & 1\end{array}\right]$

where $A^{\text {III }}=1 /\left(1+2 \cdot 2^{-2 i}\right), A^{\text {III }}=1 / \sqrt{1+3 \cdot 2^{-2 i}}, \alpha=2^{-2 i}, \beta=2^{-1}$, $\operatorname{sgn}_{1}^{i}=\operatorname{sign}\left(x_{1}^{j} \cdot x_{2}^{j}\right), \operatorname{sgn}_{2}^{i}=\operatorname{sign}\left(x_{1}^{i} \cdot x_{3}^{j}\right), \quad \operatorname{sgn}_{3}^{i}=\operatorname{sign}\left(x_{1}^{j} \cdot x_{4}^{i}\right)$

산 과정 $\left(\mathrm{Q}_{\mathrm{ZII} .1}{ }^{-1}, \mathrm{Q}_{\mathrm{ZII} .2^{-1}}, \mathrm{Q}_{\mathrm{ZI} .3}{ }^{-1}\right)$ 에 대한 분석이 표 1 과 같이 주어진다. 표 1 에 기반하여, 제안된 $\mathrm{QRD}$ 방식은 먼저 $\mathrm{QZI}_{\mathrm{ZI}}{ }^{-1}$ 을 구현할 최적의 알고리즘을 선택한다. 향상된 무선 통신 표준에서 가장 높은 우선순위는 높 은 속도를 유지하는 것이기 때문에, 하우스홀더와 다 차원 알고리즘이 두 가지 가능한 후보가 된다. 여기 서 두 알고리즘 중 하우스홀더 알고리즘보다 낮은 복 잡도로 인해서 다차원 알고리즘을 최종 선택한다. $\mathrm{QZI}^{-1}$ 에 대해서는 가장 낮은 대기시간을 갖는 다른 알고리즘들에 비해 낮은 복잡도를 보여주는 하우스홀 더 알고리즘을 선택한다. 비슷한 방법으로, 마지막 
$\mathrm{QZI}^{-1}$ 은 기븐스 회전 알고리즘을 선택한다. 제안된 $\mathrm{QRD}$ 방식은 임의의 차원의 채널 매트릭스에 적용 가 능하며, 채널 매트릭스의 차원이 커질수록 그 효과도 점진적으로 증가한다. 매트릭스 차원의 증가에 따른 제안하는 방식의 효과는 5 장 $(\mathrm{V})$ 에서 실험적으로 분 석하였다.

Table 1. Analysis of latency and computational complexity for different $4 \times 4$ QRD algorithms.

표 $\quad 1.4 \times 4 \mathrm{QRD}$ 알고리즘들의 대기시간과 연산복잡 도 분석

\begin{tabular}{|c|c|c|c|c|c|c|c|c|c|c|}
\hline \multirow{2}{*}{\multicolumn{2}{|c|}{$\begin{array}{l}\text { QRD 유형 } \\
\text { 곱셈 행렬 }\end{array}$}} & \multicolumn{3}{|c|}{$\mathrm{HH}[9]$} & \multicolumn{3}{|c|}{ MD[11] } & \multicolumn{3}{|c|}{ GR[7] } \\
\hline & & $Q_{Z Z 1.1}{ }^{-1}$ & $\mathrm{Q}_{\mathrm{ZI} \cdot 2}{ }^{-1}$ & $Q_{71.3}{ }^{-1}$ & $Q_{Z Z 1.1}{ }^{-1}$ & $\mathrm{Q}_{\mathrm{ZI2} 2}{ }^{-1}$ & $\mathrm{Q}_{\mathrm{ZI.}, 3}{ }^{-1}$ & $Q_{Z Z 11}{ }^{-1}$ & $\mathrm{Q}_{\mathrm{ZI.2} 2}{ }^{-1}$ & $Q_{71.3}{ }^{-1}$ \\
\hline \multicolumn{2}{|c|}{$\begin{array}{c}\text { 대기 시간 } \\
\text { (클락 } \\
\text { 주기) }\end{array}$} & \multicolumn{3}{|c|}{$\mathrm{N}$} & \multicolumn{3}{|c|}{$\mathrm{N}$} & \multicolumn{2}{|c|}{$2 \mathrm{~N}$} & $\mathrm{~N}$ \\
\hline \multirow{4}{*}{$\begin{array}{l}\text { 연 } \\
\text { 산 } \\
\text { 복 } \\
\text { 잡 } \\
\text { 도 }\end{array}$} & 덧셈 & $119 \mathrm{~N}$ & $35 \mathrm{~N}$ & $12 \mathrm{~N}$ & $84 \mathrm{~N}$ & $95 \mathrm{~N}$ & $6 \mathrm{~N}$ & $42 \mathrm{~N}$ & $20 \mathrm{~N}$ & $6 \mathrm{~N}$ \\
\hline & $\begin{array}{l}\text { 부호 } \\
\text { 반전 }\end{array}$ & $84 \mathrm{~N}$ & $30 \mathrm{~N}$ & $6 \mathrm{~N}$ & $84 \mathrm{~N}$ & $50 \mathrm{~N}$ & $6 \mathrm{~N}$ & $42 \mathrm{~N}$ & $20 \mathrm{~N}$ & $6 \mathrm{~N}$ \\
\hline & $\begin{array}{l}\text { 쉬프 } \\
\text { 트 }\end{array}$ & $\begin{array}{c}28 \mathrm{M}+ \\
21\end{array}$ & $\begin{array}{c}15 \mathrm{M}^{+} \\
14\end{array}$ & $3 \mathrm{M}+2$ & $14 \mathrm{M}$ & $\begin{array}{c}15 \mathrm{M}^{+} \\
15\end{array}$ & $3 \mathrm{M}$ & $10.5 \mathrm{M}$ & $5 \mathrm{M}$ & $3 \mathrm{M}$ \\
\hline & XOR & $6 \mathrm{~N}$ & $3 \mathrm{~N}$ & $\mathrm{~N}$ & $3 \mathrm{~N}$ & $3 \mathrm{~N}$ & 0 & 0 & 0 & 0 \\
\hline
\end{tabular}

\section{IV. 제 안하는 복합 $\mathrm{QR}$ 분해 구조}

제안하는 $\mathrm{QRD}$ 방식에 기반한 $4 \times 4 \mathrm{QRD}$ 구조는 그 림 1.과 같이 나타낼 수 있다. 그림에서 회색, 검정색, 흰색 사각형은 각각 다차원 코딕, 하우스홀더, 기븐스 회전 알고리즘에 기반한 코딕 모듈을 보여준다. 사각

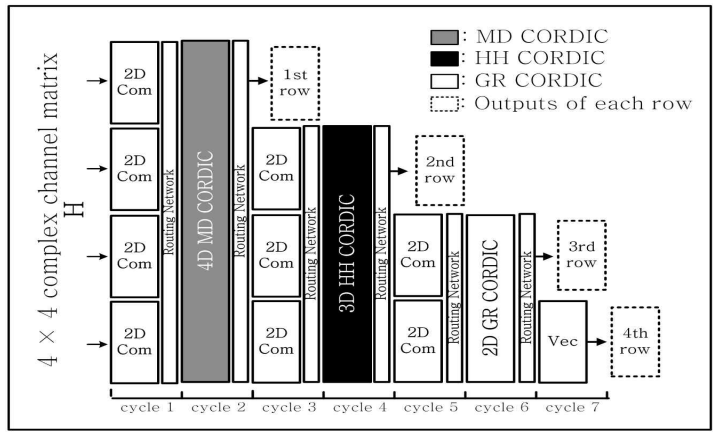

Fig. 1. The proposed $4 \times 4 \mathrm{QRD}$ architecture 그림 1. 제안하는 $4 \times 4 \mathrm{QRD}$ 구조

형 안의 숫자는 각 코딕 모듈의 차원을 의미하며, 단
위 코딕 모듈의 대기시간을 사각형 밑의 cycle로 나 타낸다. Cycle 1, 3, 5에서 행렬 $\mathrm{QRT}_{\mathrm{RT}}{ }^{-1}, \mathrm{QRT}_{2}{ }^{-1}$, $\mathrm{QRT}^{-1}{ }^{-1}$ 의 곱셈을 수행하는 compact 코딕 모듈을 Com 으로 나타낸다[12]. 3장(III)에서 분석했듯이, cycle 2, 4, 6에서 수행하는 행렬 $\mathrm{QZII.1}^{-1}, \mathrm{QZZI}^{-1}, \mathrm{QZI.}^{-1}$ 의 곱셈 은 각각 다차원 코딕, 하우스홀더, 기븐스 회전으로 구현한 코딕 모듈을 사용한다. $\mathrm{QRD}$ 과정의 마지막 단계인 cycle 7 에서, 4행 4열의 복소 성분을 실수로 전환하는 데에는 코딕 모듈의 벡터링 방식을 사용한 다. 여기서 각 코딕의 대기시간은 8 클락 주기로 정 하는데, 이는 라디안으로 표시하는 평균 잔여 각 오

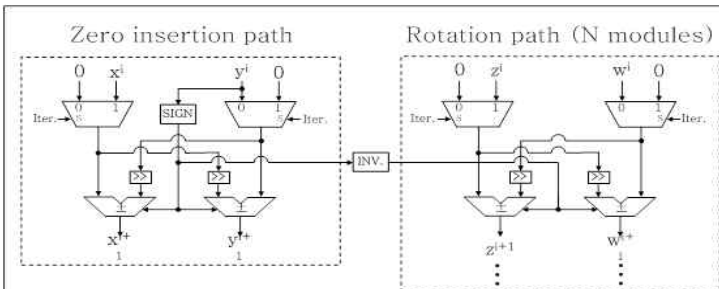

(a)

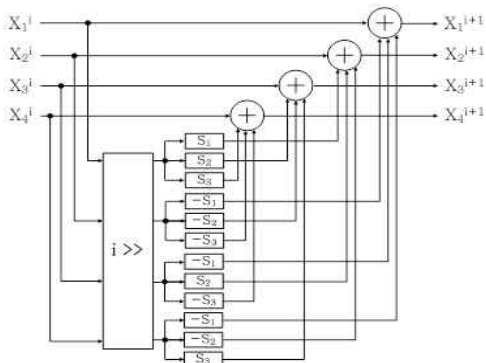

$\mathrm{S}_{1}=\operatorname{sign}\left(\mathrm{X}_{1}{ }^{\mathrm{i}} \cdot \mathrm{X}_{2}^{\mathrm{i}}\right), \mathrm{S}_{2}=\operatorname{sign}\left(\mathrm{X}_{1}^{\mathrm{i}} \cdot \mathrm{X}_{3}^{\mathrm{i}}\right), \quad \mathrm{S}_{3}=\operatorname{sign}\left(\mathrm{X}_{1}^{\mathrm{i}} \cdot \mathrm{X}_{4}^{\mathrm{i}}\right)$

(b)

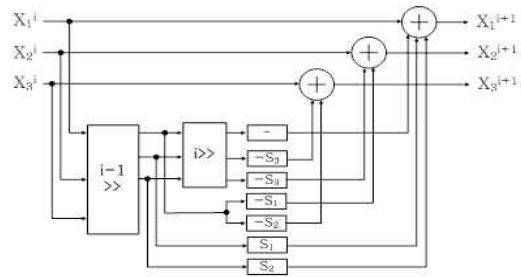

$\mathrm{S}_{1}=\operatorname{sign}\left(\mathrm{X}_{1}{ }^{\mathrm{i}} \cdot \mathrm{X}_{2}{ }^{\mathrm{i}}\right), \mathrm{S}_{2}=\operatorname{sign}\left(\mathrm{X}_{1}{ }^{\mathrm{i}} \cdot \mathrm{X}_{3}{ }^{\mathrm{i}}\right), \operatorname{Sgn}_{3}=\operatorname{sign}\left(\mathrm{X}_{2}{ }^{\mathrm{i}} \cdot \mathrm{X}_{3}{ }^{\mathrm{i}}\right)$ (c)

Fig. 2. The component of the $4 \times 4 \mathrm{QRD}$ architecture (a) N-D Compact CORDIC module (b) The basic unit in 4D MD CORDIC (c) The basic unit in 3D $\mathrm{HH}$ CORDIC

그림 2. $4 \times 4 \mathrm{QRD}$ architecture의 구성 요소 (a) N-D Compact 코딕 모듈 (b) $4 \mathrm{D}$ 다차원 코딕 기본 유 닛 (c) $3 \mathrm{D}$ 하우스홀더 코딕 기본 유닛

류를 $10^{-2}$ 보다 낮추어[13] 충분한 정확도를 갖는 결 과 값을 도출하도록 만든다. 따라서 채널 매트릭스의 
입력 중 가장 첫 입력 값에 대한 결과 값은 첫 입력 후 56 클락 주기 후에 나오며, 이후부터는 각 코딕 모듈의 연결 부분에 적용된 파이프라이닝으로 인해 결과 값을 대응되는 채널 매트릭스의 입력 후 8 클락 주기 후에 얻을 수 있다.

제안하는 구조의 각 구성 요소는 그림 2.에 나타내 었다. 그림 2 (a)의 N-D compact 코딕은 zero insertion path와 rotation path으로 나뉜다. 여기서 zero insertion path는 $(5-\mathrm{N})$ 번째 열의 zero insertion 과정을 수행하고, rotation path는 나머지 $(\mathrm{N}-1)$ 부분 에 대한 행렬 곱을 수행한다. $4 \mathrm{D}$ 다차원 코딕과 $3 \mathrm{D}$ 하우스홀더 코딕의 기본 단위 모듈은 그림 2. (b)와 (c)에 각각 제시하였다. 기본 모듈은 같은 열의 성분 대하여 동작을 수행하며, 실수와 허수 부분을 독립적 으로 실행한다. $4 \mathrm{D}$ 다차원 코딕과 $3 \mathrm{D}$ 하우스홀더 코 딕 모듈은 전체 7 개(=1(첫 번째 열) +3 (첫 번째 열을 제외한 나머지 3 열의 실수 성분) +3 (나머지 3 열의 허 수 성분))와 5 개 $(=1$ (두 번째 열) +2 (첫 번째, 두 번째 열을 제외한 2 열의 실수 성분 $)+2$ (첫 번째, 두 번째 열 을 제외한 2 열의 허수 성분))의 기본 모듈을 각각 필 요로 한다.

Table 2. Comparison with the conventional $4 \times 4 \mathrm{QR}$ decomposition architectures.

표 2. 기존 $4 \times 4 \mathrm{QR}$ 분해 구조와의 비교

\begin{tabular}{|c|c|c|c|c|}
\hline & [6] & [8] & [10] & 제안구조 \\
\hline 공정 $(\mu \mathrm{m})$ & 0.13 & 0.18 & 0.13 & 0.13 \\
\hline QRD 유형 & GR & GR & $\mathrm{HH}$ & $\mathrm{MD}, \mathrm{HH}, \mathrm{GR}$ \\
\hline $\begin{array}{c}\text { 대기시간 } \\
\text { (클락주기) }\end{array}$ & 67 & 4 & 8 & 8 \\
\hline $\begin{array}{c}\text { 최대 동작 주파수 } \\
(\mathrm{MHz})\end{array}$ & 125 & 100 & 215 & 250 \\
\hline $\begin{array}{c}\text { Gate } \\
\text { count } \\
\text { (2nnd) }\end{array}$ & $54 \mathrm{~K}$ & $111 \mathrm{~K}$ & $83.7 \mathrm{~K}$ & $66.1 \mathrm{~K}$ \\
\hline $\begin{array}{c}\text { QRD } \\
\text { Rate[8] }\end{array}$ & $1 / 536 n$ & $1 / 40 \mathrm{n}$ & $1 / 37.2 \mathrm{n}$ & $1 / 32 \mathrm{n}$ \\
\hline $\begin{array}{c}\text { QAR } \\
\text { (QRD } \\
\text { Rate/Gatecount) }\end{array}$ & 34.5 & 225.2 & 321.1 & 472.7 \\
\hline $\begin{array}{c}\text { 전력 소모(mW) } \\
@ 215 \mathrm{MHz}\end{array}$ & N/A & N/A & 26.7 & 19.2 \\
\hline
\end{tabular}

\section{V. 실험 결과}

표 2.에서는 제안하는 구조와 기존 고속 $\mathrm{QRD}$ 구조 를 비교하였다. 제안하는 $\mathrm{QRD}$ 방식을 적용함으로써, 제안하는 구조의 gate count가 더 적은 것을 볼 수
있다[10]. 여기서, 제안하는 구조를 적용함으로써 줄어 든 최대 지연시간 때문에 동작 주파수 또한 증가한 다. [10]과 제안하는 구조에 대해, Primtime-PX[14]를 사용하여 동작주파수 $215 \mathrm{MHz}$, 공급 전압 $1.2 \mathrm{~V}$ 에서 전력 소모를 측정하였다. BER 성능 측면에서는, $\mathrm{QRM}-\mathrm{MLD}$ 기반 64-QAM $\mathrm{MIMO}$ 검출기 $(\mathrm{M}=16)$ 에 적용하였을 때, 제안하는 구조가 [10]와 $15 \mathrm{~dB}$ 에서 $30 \mathrm{~dB}$ 까지 비슷하였다. BER 그래프에서 제안하는 구 조와 [10]의 $\mathrm{Eb} / \mathrm{N} 0$ 축 최대 차이도 $0.1 \mathrm{~dB}$ 미만이었 다. 결과적으로, 제안하는 구조는 기존 연구들에 비해 작은 성능 저하로 최고의 $\mathrm{QRD}$ rate Area Ratio(QAR)을 보인다.

표 3에서는 제안하는 구조의 연산 복잡도를 하우스 홀더, 다차원 코딕 방법의 연산 복잡도와 높은 차원 의 행렬에서 비교하였다. 그리고 두 가지 가장 복잡 한 연산에 대한 (연산량/반복횟수)를 나타냈는데, 특 별히 가장 복잡한 덧셈 연산에서 채널 매트릭스 차원 의 증가에 따라 그 감소 비율이 매우 큼을 알 수 있 다. $8 \times 8$ 행렬의 경우, 제안하는 구조를 적용했을 때, 하우스홀더와 다차원 코딕 방식과 비교하여 각각 $45.3 \%$ 와 $29.5 \%$ 의 덧셈연산이 줄어든다. 최근의 $4 \mathrm{G}$ 무선 통신 표준은 8 개의 복수 안테나 지원을 필요로 하기 때문에, 제안하는 구조는 미래의 휴대용 기기에 더욱 많이 적용될 것으로 보인다.

Table 3. Computational complexity comparisons for diferent QR decomposition architectures.

표 3. 다양한 $\mathrm{QR}$ 분해 구조들의 연산 복잡도 비교

\begin{tabular}{|c|c|c|c|c|c|c|c|}
\hline \multicolumn{2}{|c|}{ QRD 알고리즘 } & \multicolumn{2}{|c|}{$\mathrm{HH}[9]$} & \multicolumn{2}{|c|}{$\mathrm{MD}[11]$} & \multicolumn{2}{|c|}{$\begin{array}{c}\text { 제안 } \\
\text { 알고리즘 }\end{array}$} \\
\hline \multicolumn{2}{|c|}{ 연산 유형 } & 덧셈 & $\begin{array}{l}\text { 부호 } \\
\text { 반전 }\end{array}$ & 덧셈 & $\begin{array}{l}\text { 부호 } \\
\text { 반젖 }\end{array}$ & 덧셈 & $\begin{array}{l}\text { 부호 } \\
\text { 반전 }\end{array}$ \\
\hline \multirow{4}{*}{$\begin{array}{c}\text { 연산량/ } \\
\text { 반복횟수 } \\
\text { (비율,\%) }\end{array}$} & $5 \times 5$ & $\begin{array}{c}396 \\
(100)\end{array}$ & $\begin{array}{c}344 \\
(100)\end{array}$ & $\begin{array}{c}486 \\
(123)\end{array}$ & $\begin{array}{c}342 \\
(99.4)\end{array}$ & $\begin{array}{c}294 \\
(74.2)\end{array}$ & $\begin{array}{c}278 \\
(80.8)\end{array}$ \\
\hline & $6 \times 6$ & $\begin{array}{c}864 \\
(100)\end{array}$ & $\begin{array}{c}564 \\
(100)\end{array}$ & $\begin{array}{l}1077 \\
(125)\end{array}$ & $\begin{array}{c}690 \\
(122)\end{array}$ & $\begin{array}{c}561 \\
(64.9)\end{array}$ & $\begin{array}{c}518 \\
(91.8)\end{array}$ \\
\hline & $7 \times 7$ & $\begin{array}{l}1599 \\
(100)\end{array}$ & $\begin{array}{c}954 \\
(100)\end{array}$ & $\begin{array}{l}1739 \\
(109)\end{array}$ & $\begin{array}{l}1154 \\
(121)\end{array}$ & $\begin{array}{c}959 \\
(59.9)\end{array}$ & $\begin{array}{c}894 \\
(93.7)\end{array}$ \\
\hline & $8 \times 8$ & $\begin{array}{l}2687 \\
(100)\end{array}$ & $\begin{array}{l}1584 \\
(100)\end{array}$ & $\begin{array}{c}2430 \\
(90.4)\end{array}$ & $\begin{array}{l}1720 \\
(109)\end{array}$ & $\begin{array}{c}1470 \\
(54.7)\end{array}$ & $\begin{array}{c}1400 \\
(88.3)\end{array}$ \\
\hline
\end{tabular}

\section{VI. 결론}

본 논문에서는, 다양한 코딕 기반 $\mathrm{QRD}$ 알고리즘의 최적의 조합을 가능하게 하는 제안된 $\mathrm{QRD}$ 방법을 통 한 저 복잡도 고속 $\mathrm{QRD}$ 구조를 제시하였다. 제안된 
접근 방식은 임의의 차원의 채널 매트릭스에 적용가 능하다. 본 논문의 $\mathrm{QRD}$ 설계는 삼성 $0.13 \mu \mathrm{m}$ 공정으 로 구현되었으며, 제안된 구조는 $47 \%$ 의 $\mathrm{QAR}(\mathrm{QRD}$ Rate/Gate count) 향상과 $28.1 \%$ 의 전력 절감효과를 갖는다. 실험결과 $8 \times 8$ 복소행렬의 $\mathrm{QRD}$ 에 대해서 기존의 하우스홀더 방법과 비교하여 $45.3 \%$ 의 덧셈연 산이 감소하였다. 본 논문에서 제시한 아이디어는 향 상된 무선 통신 표준의 $\mathrm{QRD}$ 구현에 기여할 것으로 사료된다.

\section{References}

[1] Wireless LAN Medium Access Control (MAC) and Physical Layer (PHY) Specifications: Enhancements for Very High Throughput for Operation in Bands Below 6GHz, IEEE P802.11ac/D 1.0 Standard, Jan. 2011.

[2] S. Haykin, Communication Systems, 4th ed. New York: Wiley, 2000.

[3] M. Shabany, P. Gulak, "A 675 Mbps, 4x4 64-QAM K-Best MIMO Detector in 0.13 um CMOS," IEEE Trans. Very Large Scale Integr.(VLSI) Syst., vol. 20, no. 1, Jan. 2012.

[4] M. Lei, K. Dickson, J. McAllister, and J. Mc Canny, "QR decomposition-based matrix inversion for high performance embedded mimo receivers," Signal Processing, IEEE Transactions on, vol. 59, no. 4, pp. 1858-1867, 2011.

[5] P. K. Meher, J. Valls, J. Tso-Bing, K. Sridharan, and K. Maharatna, "50 years of cordic: Algorithms, architectures, and applications," Circuits and Systems I: Regular Papers, IEEE Transactions on, vol. 56, no. 9, pp. 1893-1907, 2009.

[6] P. Luethi, A. Burg, S. Haene, D. Perels, N. Felber, and W. Fichtner, "VLSI Implementation of a High-Speed Iterative Sorted MMSE QR Decomposition," in Proc. IEEE ISCAS 2007, pp. 1421-1424, May 2007.

[7] Min-Woo Lee, Ji-Hwan Yoon, and Jongsun Park, "High-Speed Tournament Givens Rotation-based QR Decomposition Architecture for MIMO Receiver," in Circuit and Systems (ISCAS), 2012 IEEE international Symposium on, May 2012.

[8] Z. Huang, P. Tsai, "Efficient Implementation of
QR Decomposition for Gigabit MIMO-OFDM Systems," Circuits and Systems I: Regular Papers, IEEE Transactions on , vol.58, no.10, pp.2531-2542, Oct. 2011.

[9] J. Delosme and S. Hsiao, "Householder CORDIC algorithms," IEEE Trans. Comput., vol. 44, no. 8, pp. 990-1001, Aug. 1995.

[10] Kurniawan. I. H., Ji-Hwan Yoon, and Jongsun Park, "Multidimensional Householder based high-speed QR decomposition architecture for MIMO receivers," in Circuit and Systems (ISCAS), 2013 IEEE international Symposium on, May 2013.

[11] S. Hisao, J. Delosme, Multi-dimensional CORDIC algorithms, 1993.

[12] Jae-Woong Han, Young-Beom Jang, “A Residual Frequency Offset Synchronization Scheme Using a Simplified CORDIC Algorithm in OFDM Systems," Communications TheoryWorkshop,2009.AusCTW2 009.Australian , vol., no., pp.67,70, 4-7 Feb. 2009 .

[13] C. Wu, A. Wu, "Modified vector rotational CORDIC (MVR-CORDIC) algorithm and architecture," CircuitsandSystemsII: Analog and Digital Signal Processing, IEEE Transactions on, vol.48, no.6, pp.548,561, Jun 2001.

[14] Synopsys PrimeTime User's Manual, http://www.synopsys.com

\section{BIOGRAPHY}

Shin Dongyeob (Student Member)

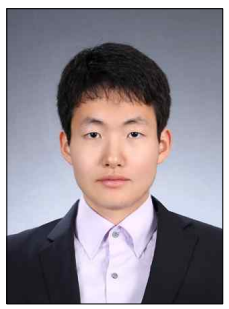
2013 : B.S. degree in Electrical Engineering, Korea University. 2013 : Graduate student toward M.S and Ph.D. degree in Electrical Engineering, Korea University. 
Kim Chulwoo (Member)

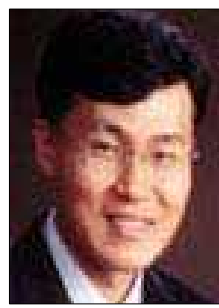

1994 : B.S. degree in Electrical

Engineering, Korea University.

1996 : M.S. degree in Electrical

Engineering, Korea University.

2001 : Ph.D. degree in

Electrical and Computer

Engineering, University of

Illinois at Urbana-Champaign, US.

2002 : Professor, Department of Electrical

Engineering, Korea University.

Park Jongsun (Member)

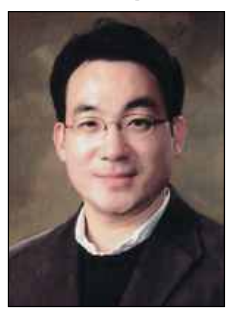

2000 : B.S. degree in Electrical

Engineering, Korea University.

2005 : M.S. and Ph.D. degree in

Electrical and Computer

Engineering, Purdue University,

US.

2005 2008 : Marvell

Semiconductors Staff Design Engineer.

2008 : Associate Professor, Department of

Electrical Engineering, Korea University. 